\title{
Hiv-2 interaction with cell receptors
}

\begin{abstract}
Although sharing several properties, human immunodeficiency virus 1 and 2 (HIV-1 and HIV-2) have shown some important differences in vivo. A significant amount of data suggest that HIV-2 is in general less virulent than HIV-1. This reduced virulence is revealed by a longer asymptomatic period, a minor T-cell depletion and lower viral load. Due to its inherently attenuated phenotype, the study of HIV-2 infection constitutes an exceptionally good model to understand virologic and pathogenic mechanisms that enable the human host to cope with an HIV infection for such a long period of time and may help to learn more about HIV-1 pathogenesis, and to viral-cell interactions, opening to new strategies to vaccines or therapeutic design. The molecular mechanisms underlying this reduced virulence are far from being fully characterized or even identified. In this review the contribution of virus-cell interactions for this phenotype will be discussed with particular emphasis in the events involved in binding of envelope glycoproteins to cell co receptors.
\end{abstract}

Keywords: HIV-2, Cell Receptors, Chemokine Receptors, Viral Entry, Pathogenesis, Envelope Glycoprotein's, Apoptosis
Volume 2 Issue 3 - 2015

\author{
Jose Miguel Azevedo- Pereira ${ }^{1,2}$ \\ 'Host Pathogen Interaction Unit, Instituto de Investigacao \\ do Medicamento (iMed.ULisboa), Faculdade de Farmacia, \\ Universidade de Lisboa, Portugal \\ ${ }^{2}$ Instituto de Medicina Molecular (IMM), Faculdade de Medicina, \\ Universidade de Lisboa, Portugal
}

Correspondence: Jose Miguel Azevedo Pereira, Host-Pathogen Interaction Unit, Research Institute for Medicines, Instituto de Investigacao do Medicamento (iMed.ULisboa), Faculty of Pharmacy, University of Lisbon, Lisboa, Portugal, Tel 35I-2I7946400, Fax 35I-2I-79342I 2 Email miguel.pereira@ff.ul.pt

Received: March 03, 2015 | Published: April 03, 2015
Abbreviations: HIV, Human immunodeficiency Virus; AIDS, Acquired immunodeficiency syndrome; ENV, Envelope; SU, Surface glycoprotein; TM, Transmembrane glycoprotein; V1/ V2, Variable regions 1 and 2; V3, Variable region 3; CCR5, CC Chemokine receptor 5; CCR8, CC Chemokine receptor 8; CXCR4, CXC Chemokine receptor 4; GALT, gut-associated lymphoid tissue; LFA-1, lymphocyte function-associated antigen $1 ; \alpha 4 \beta 7$, alpha4beta7 integrin

\section{Introduction}

Human immunodeficiency virus 2 (HIV-2) is less virulent in vivo compared to human immunodeficiency virus 1 (HIV-1). This reduced virulence is revealed by a longer asymptomatic period, minor T-cell depletion and lower viral load. The basic molecular mechanisms, (both viral and cellular) underlying this reduced virulence is far from being fully characterized or even identified. We hypothesize that the way HIV-2 interacts with cellular receptors contributes decidedly to this lower virulence and enables the preservation of host immune function for a longer period of time. In this review the main characteristics of HIV-2 interaction with cellular receptor are focused and the major differences to HIV-1 are highlighted.

\section{Discussion}

Disease progression in HIV infection is accompanied by a continuous and irreversible decline of CD4+ T-lymphocyte numbers, predisposing the host to life-threatening opportunistic infections. Understanding the mechanism of CD4 cell loss in HIV infection is essential to understand viral pathogenesis and for the development of effective therapeutic strategies. In this regard, HIV-1 and HIV-2 show some important differences in vivo. Although sharing the same transmission routes, HIV-2 infection is in general characterized by:

\section{i. A longer asymptomatic stage}

\section{ii. A lower viral load}

iii. A minor T-cell depletion and consequently to a lower rate of disease progression (reviewed $\mathrm{in}^{1,2}$ ).

In fact, despite both viruses are linked to the onset of Acquired Immunodeficiency Syndrome (AIDS), HIV-2 infection has little impact on survival of infected individuals..$^{3-5}$ These observations lead to the assumption that virtually all HIV-2 infected patients fit in a definition of "long-term non-progressors" or "elite-controlers", a condition that appropriately define those rare HIV-1 infected individuals. ${ }^{6}$ That remains healthy for several decades without any antiretroviral therapy, with undetectable plasma viral load and CD4+ cell counts above 500 cells $/ \mu \mathrm{L}$.

The initial events that lead to viral entry have been related to several important characteristics of HIV. Modifications, either in cellular receptors or in viral receptor-interacting glycoproteins, lead inevitably to major viral phenotype changes that include adjustment of cell tropism, altered replicative fitness, different abilities to induce T-cell depletion or to escape neutralizing antibodies and, ultimately, to unique pathogenic characteristics. In the most favored model(reviewed in ${ }^{7,8}$ ), HIV entry begins with a specific interaction between the virion heterodimeric Env complex, formed by surface (SU) and trans membrane (TM) glycoproteins, and two cellular proteins: CD4 and a chemokine receptor (referred as co receptor). Several of these receptors have been described in vitro as potential cofactors for HIV entry into target cells. However, despite this extensive range of molecules that could act as viral co receptor, CCR5 and CXCR4 are considered as the main coreceptors for HIV-1 and the only that has a well-defined role in HIV pathogenesis..$^{9,10}$

Based on our previous work we hypothesize that one of the factors that greatly contributes to the lower virulence of HIV-2 is related with the efficiency with which HIV-2 uses cellular receptors to interact with target cells. This hypothesis stems from our previous observation regarding CD4-independent infection ${ }^{11}$ and non-usage of neither CCR5 nor CXCR4 by HIV-2 strains. ${ }^{12-15}$

These observations clearly support the notion that HIV-2 and HIV1 use cellular receptors in different ways with direct implications in viral pathogenesis. ${ }^{1}$ Furthermore, the molecular determinants of coreceptor engagement seem also to be different in HIV-1 and HIV-2. While in HIV-1 the variable region 3 (V3) of Env SU glycoprotein is the major determinant of coreceptor usage, ${ }^{16-19}$ in HIV-2 the contribution of $\mathrm{V} 3$ region seems to be much less defined. In this regard, we recently show ${ }^{12}$ that the use of CCR8 as coreceptor and the switch from CCR8 to CCR5 or CCR5/CXCR4 usage is determined by amino acids located in variable regions 1 and 2 (V1/V2) of HIV-2 SU envelope glycoprotein. 
The usage of cellular receptors by HIV enables the virus to overcome the plasma cell membrane barrier and permits the delivery of its genetic material into host cell cytoplasm. In addition, the interactions between HIV Env glycoproteins with coreceptor and the misappropriation of chemokine receptor function, triggers the activation of several intracellular pathways that leads to an array of physiological changes in infected cell (e.g. chemo taxis, proliferation, cytokine secretion, differentiation). Although receptor signaling is not required for coreceptor function, ${ }^{20-22}$ these chemokine signaling cascades in vivo may prepare the target cells for viral replication and conceivably they could be responsible for some of the cellular responses to the virus. ${ }^{23}$

One of them is the induction of apoptotic signaling. This HIV mediated apoptosis is largely responsible for the gradual depletion of CD4+ T-lymphocytes, although other mechanisms have also been described. Although HIV encodes several apoptogenic proteins (e.g. Env, Tat, Vpr, Vpu and Nef), Env interaction with cellular receptors has been consistently referred as a principal mechanism of T-cell apoptosis. ${ }^{23}$ The mechanisms underlying this programmed cell death are directly dependent on Env glycoprotein and the way it interacts with cell receptors (reviewed $\mathrm{in}^{24}$ ). Accordingly, different Env proteins with different coreceptors usage profiles induce different signaling pathways and conceivably could induce different levels of apoptosis in infected and bystander non-infected cells. ${ }^{25}$

In addition, it was shown ${ }^{26}$ that HIV-1 is able to bind and signal through the integrin alpha4-beta7 ( $\alpha 4 \beta 7)$ that mediates the migration of lymphocytes to gut-associated lymphoid tissue (GALT). Regardless of the route of transmission, HIV-1 rapidly establishes infection in GALT, converting this lymphoid tissue in the principal site of viral replication, followed by a severe CD4+ T-cell depletion. ${ }^{27,28}$ The slower rate of CD4+ T-cell decline in HIV-2 infection may reflect differences during the initial phase of HIV-1 and HIV-2 infections and directly related with GALT infection. Since HIV-2 induces a naturally less pathogenic infection, an obvious question arises: is HIV-2 interaction with $\alpha 4 \beta 7$ as efficient as HIV-1? And does this interaction leads to the same sort of cellular signaling such as the activation of LFA-1, an integrin that facilitates cell-to-cell transfer of HIV through the induction of virological synapses ${ }^{29}$ ? Although these effects are hypothetically viral strain-dependent, we hypothesize that HIV-2 may interact with $\alpha 4 \beta 7$ less efficiently leading to a minor colonization of the GALT and hence to a lower depletion of CD4+ T-lymphocytes.

\section{Conclusion}

Very few data exists about the cellular consequences of HIV-2 interaction with cellular receptors. The plasticity of HIV-2 Env SU glycoprotein, leading to an unusual profile of coreceptors usage, could allow HIV-2 to "accidentally" infect inappropriate cell populations or induce less-than-optimal signaling after interaction with cellular receptors and thus be responsible for abortive infections, lower viral yields and minor T-cell apoptosis. It is thus important to assess the outcome of these interactions since them could explain the attenuated disease of HIV-2. Further studies are of utmost importance focusing in particular viruses obtained from asymptomatic individuals, since the main differences between HIV-1 and HIV-2 pathogenesis are particularly striking during this stage where a significantly slower clinical progression and lower CD4+ T-cell depletion are observed.

\section{Acknowledgements}

This work was supported by a grant from Fundação para a Ciência e Tecnologia and Ministério da Saúde de Portugal (Grant VIH/ SAU/0006/2011).

\section{Conflicts of interest}

None.

\section{References}

1. Azevedo-Pereira JM, Santos-Costa Q, Moniz-Pereira J HIV-2 infection and chemokine receptors usage - clues to reduced virulence of HIV-2. Curr HIV Res. 2005;3(1):3-16.

2. Reeves JD, Doms RW. Human immunodeficiency virus type 2. J Gen Virol. 2002;83(Pt 6):1253-1265.

3. Whittle H, Morris J, Todd J, et al. HIV-2-infected patients survive longer than HIV-1-infected patients. AIDS. 1994;8(11):1617-1620

4. Poulsen AG, Aaby P, Larsen O, et al. 9-year HIV-2-associated mortality in an urban community in Bissau, west Africa. Lancet. 1997;349(9056):911-914.

5. Marlink R, Kanki P, Thior I, et al. Reduced rate of disease development after HIV-2 infection as compared to HIV-1. Science. 1994;265(5178):1587-1590.

6. Lambotte O, Boufassa F, Madec Y, et al. HIV controllers: a homogeneous group of HIV-1-infected patients with spontaneous control of viral replication. Clin Infect Dis. 2005;41(7):1053-1056.

7. Clapham PR, McKnight A. Cell surface receptors, virus entry and tropism of primate lentiviruses. J Gen Virol. 2002;83(Pt 8):1809-1829.

8. Doms RW, Trono D. The plasma membrane as a combat zone in the HIV battlefield. Genes Dev. 2000;14(21):2677-2688.

9. Simmons G, Reeves JD, Hibbitts S, et al. Co-receptor use by HIV and inhibition of HIV infection by chemokine receptor ligands. Immunol Rev. 2000;177:112-126.

10. Zhang YJ, Dragic T, Cao Y, et al. Use of coreceptors other than CCR5 by non-syncytium-inducing adult and pediatric isolates of human immunodeficiency virus type 1 is rare in vitro. $J$ Virol. 1998;72(11):9337-9344.

11. Reeves JD, Hibbitts S, Simmons G, et al. Primary human immunodeficiency virus type 2 (HIV-2) isolates infect CD4-negative cells via CCR5 and CXCR4: comparison with HIV-1 and simian immunodeficiency virus and relevance to cell tropism in vivo. $J$ Virol. 1999;73(9):7795-7804.

12. Santos-Costa Q, Lopes M, Lopes M, et al. HIV-2 interaction with cell coreceptors: amino acids within the V1/V2 region of viral envelope are determinant for CCR8, CCR5 and CXCR4 usage. Retrovirology. 2014;11(1):99.

13. Santos-Costa Q, Parreira R, Moniz-Pereira J, et al. Molecular characterization of the env gene of two CCR5/CXCR4-independent human immunodeficiency 2 primary isolates. $J$ Med Virol. 2009;81(11):1869-1881.

14. Santos-Costa Q, Mansinho K, Moniz-Pereira J, et al. Characterization of HIV-2 chimeric viruses unable to use CCR5 and CXCR4 coreceptors. Virus Res. 2009;142(1-2):41-50.

15. Azevedo-Pereira JM, Santos-Costa Q, Mansinho K, et al. Identification and characterization of HIV-2 strains obtained from asymptomatic patients that do not use CCR5 or CXCR4 coreceptors. Virology. 2003;313(1):136-146

16. Cho MW, Lee MK, Carney MC, et al. Identification of determinants on a dualtropic human immunodeficiency virus type 1 envelope glycoprotein that confer usage of CXCR4. J Virol. 1998;72(3):2509-2515.

17. Hoffman TL, Doms RW. HIV-1 envelope determinants for cell tropism and chemokine receptor use. Mol Membr Biol. 1999;16(1):57-65.

18. Hu Q, Trent JO, Tomaras GD, et al. Identification of ENV determinants in V3 that influence the molecular anatomy of CCR5 utilization. $J \mathrm{Mol}$ Biol. 2000;302(2):359-375. 
19. Smyth RJ, Yi Y, Singh A, et al. Determinants of entry cofactor utilization and tropism in a dualtropic human immunodeficiency virus type 1 primary isolate. J Virol. 1998;72(5):4478-4484.

20. Aramori I, Ferguson SS, Bieniasz PD, et al. Molecular mechanism of desensitization of the chemokine receptor CCR-5: receptor signaling and internalization are dissociable from its role as an HIV-1 co-receptor. EMBO J. 1997;16:4606-4616.

21. Farzan M, Choe H, Martin KA, et al. HIV-1 entry and macrophage inflammatory protein-1beta-mediated signaling are independent functions of the chemokine receptor CCR5. J Biol Chem. 1997;272:6854-6857.

22. Gosling J, Monteclaro FS, Atchison RE, et al. Molecular uncoupling of $\mathrm{C}-\mathrm{C}$ chemokine receptor 5-induced chemotaxis and signal transduction from HIV-1 coreceptor activity. Proc Natl Acad Sci USA. 1997;94:5061-5066.

23. Ameisen JC, Lelièvre J-D, Pleskoff $\mathrm{O}$. HIV/host interactions: new lessons from the Red Queen's country. AIDS. 2002;16Supp14:S25-S31.

24. Ahr B, Robert-Hebmann V, Devaux C, et al. Apoptosis of uninfected cells induced by HIV envelope glycoproteins. Retrovirology. 2004;1:12.
25. Jekle A, Keppler OT, de Clercq E, et al. In vivo evolution of human immunodeficiency virus type 1 toward increased pathogenicity through CXCR4-mediated killing of uninfected CD4 T cells. J Virol. 2003;77(10):5846-5854

26. Arthos J, Cicala C, Martinelli E, et al. HIV-1 envelope protein binds to and signals through integrin alpha4beta7, the gut mucosal homing receptor for peripheral T cells. Nat Immunol. 2008;9(3):301-309.

27. Brenchley JM, Schacker TW, Ruff LE, et al. CD4+ T cell depletion during all stages of HIV disease occurs predominantly in the gastrointestinal tract. J Exp Med. 2004;200(6):749-759.

28. Guadalupe M, Reay E, Sankaran S, et al. Severe CD4+ T-cell depletion in gut lymphoid tissue during primary human immunodeficiency virus type 1 infection and substantial delay in restoration following highly active antiretroviral therapy. $J$ Virol. 2003;77(21):11708-11717.

29. Jolly C, Kashefi K, Hollinshead M, et al. HIV-1 cell to cell transfer across an Env-induced, actin-dependent synapse. $J$ Exp Med. 2004;199(2):283-293. 